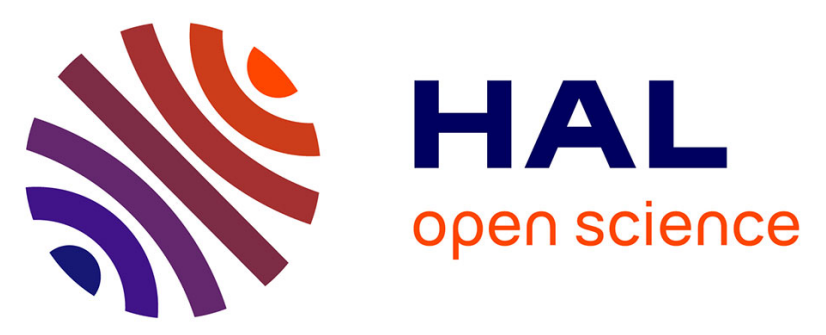

\title{
SimROS: a simulator for the design of HF surface wave radar. Application to maritime target detection
} Alain Reineix, Christophe Guiffaut, Nicolas Bourey, Muriel Darces, Marc Hélier, Sébastien Reynaud, Nicolas Ticaud, Philippe Dorey, Gilbert Auffray, Michel Menelle, et al.

\section{To cite this version:}

Alain Reineix, Christophe Guiffaut, Nicolas Bourey, Muriel Darces, Marc Hélier, et al.. SimROS: a simulator for the design of HF surface wave radar. Application to maritime target detection. 17th European Radar ConferenceConference (EuRAD) 2020, Jan 2021, Utrecht, Netherlands. 10.1109/EuRAD48048.2021.00037 . hal-03371280

\section{HAL Id: hal-03371280 \\ https://hal.sorbonne-universite.fr/hal-03371280}

Submitted on 8 Oct 2021

HAL is a multi-disciplinary open access archive for the deposit and dissemination of scientific research documents, whether they are published or not. The documents may come from teaching and research institutions in France or abroad, or from public or private research centers.
L'archive ouverte pluridisciplinaire HAL, est destinée au dépôt et à la diffusion de documents scientifiques de niveau recherche, publiés ou non, émanant des établissements d'enseignement et de recherche français ou étrangers, des laboratoires publics ou privés. 


\title{
SimROS: a simulator for the design of HF surface wave radar - Application to maritime target detection.
}

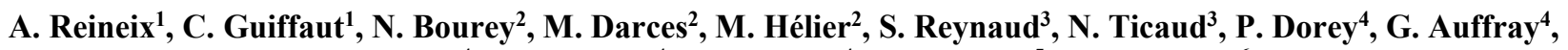

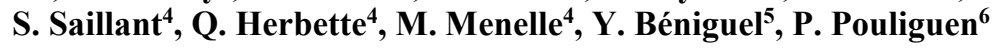 \\ ${ }^{1}$ Institut XLIM, UMR 6172, Université de Limoges, 87000 Limoges, France \\ ${ }^{2}$ Sorbonne University, CNRS, Group of electrical engineering - Paris, 75252, Paris, France \\ University of Paris-Saclay, Centrale Supélec, CNRS, Group of electrical engineering - Paris, 91192, Gif-sur-Yvette, France \\ ${ }^{3}$ CISTEME, Centre d'Ingénierie des Systèmes en Télécommunications, Electromagnétisme et Electronique, Limoges, \\ France \\ ${ }^{4}$ ONERA, Unité Radar Basse Fréquence, DEMR, 91761 PALAISEAU, France \\ 5 IEEA, 92400 Courbevoie, France \\ ${ }^{6}$ Agence de l'innovation de défense, Balard, 75509 Paris, France
}

\begin{abstract}
High Frequency (HF) radars allow the surveillance of wide zones at large distance. Their design and performance analysis require a numerical tool able to represent complex environments to model the wave propagation in realistic conditions. Particularly, in the framework of maritime surveillance, sea state model as well as sea clutter need to be considered to evaluate the radar link budget.
\end{abstract}

Keywords - HF radar, clutter, range/doppler image, reciprocity principle, link budget.

\section{INTRODUCTION}

This paper deals with a HF surface wave radar simulator. Such a radar is well suited for coastal surveillance which is mandatory for each maritime country, according to international regulation. This maritime region is called the EEZ (Exclusive Economic Zone). As the French EEZ spreads out over more than $300 \mathrm{~km}$ away from the coasts, the usual microwave radars are not operational to cover such a distance. The earth curvature requires a solution based on a non-line-ofsight propagation. It appears that the HF radar is more appropriate for such application. Two propagation modes can be used. The first one is the sky wave mode that exploits the HF wave reflection on the ionospheric layers. This mode is very efficient for very long-range detection but shows a blind zone in the EEZ. The second mode corresponds to the surface wave mode propagation that operates from some kilometres to several hundreds of kilometres. This mode will be chosen for the proposed application.

The software tool which has been developed includes several modules which are chained in an efficient way in order to obtain a budget link and a probability of detection of a maritime target. Few similar codes are available in the open literature [1].

The different modules are presented in the paper. These are the Graphical User Interface, the antenna module, the propagation tool, the radar cross section calculation and the signal processing. Some of the modules are duplicated depending on the input data available.
The objective of the proposed simulator is to provide the users, in a reasonable time (several minutes), radar performance indicators in realistic operating conditions.

\section{SIMROS CAPABILITY VIA ITS HMI INTERFACE}

The main features of the Graphical User Interface (GUI) are presented in this section.

\section{A. Global overview of the developed simulator}

The GUI presented in Fig. 1. allows defining the simulation parameters via several tabs: the definition of the sea area and its characteristics, the definition of the winds, the configuration and placement of the antennas, the characteristics of the target(s), the waveforms and the signal processing to be carried out...

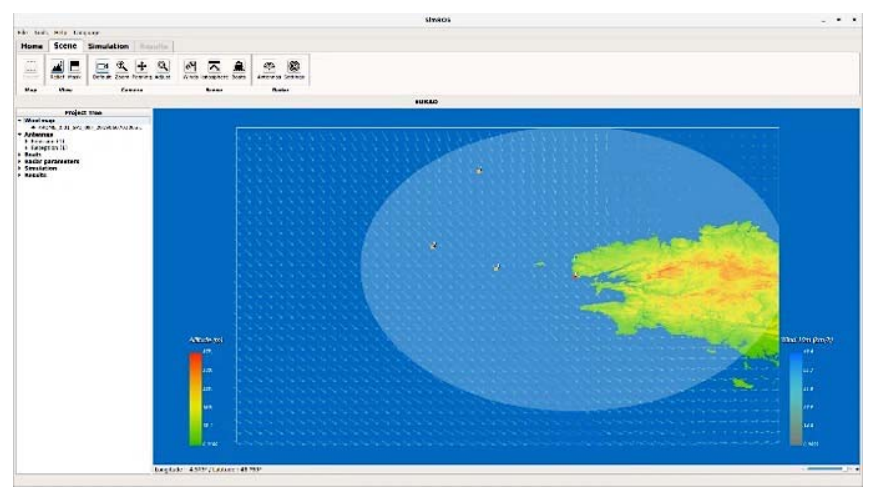

Fig. 1: HMI HF surface wave radar

Once all the blocks have been initialised, the simulation of the complete radar link can be launched, and the results viewed from the left "tree-view".

\section{B. Description of the maritime propagation environment}

The propagation solvers will take as an input a wind map (strength and direction) as the ones provided by Météo France. The maritime environment will be modelled by sea zones (named patches) whose lateral dimensions will be adjustable according to the characteristics of decorrelation of the sea state. 
The collection of patches, each of them with dedicated characteristics linked to the spectrum (for example JONSWAP spectrum), will be chained to calculate the transmitted field and the sea clutter noise. The connection between patches is performed by filtering the surfaces at the edges, so that a full maritime scene can be shaped. The example of the connection of 5 sea patches $(600 \mathrm{~m}$ by $600 \mathrm{~m}$ each) is illustrated in Fig. 2 :

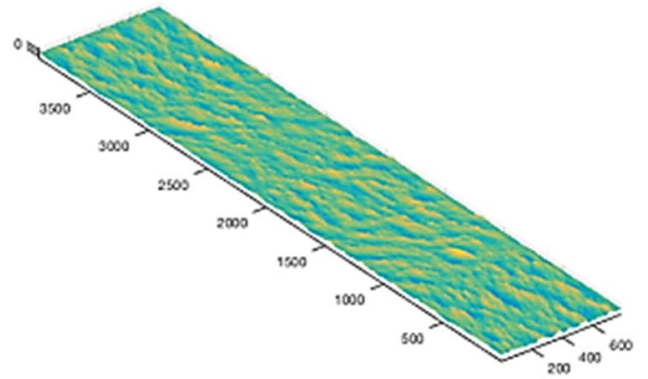

Fig. 2: Connection of 5 sea patches independently defined according to the JONSWAP spectrum

Depending on the defined sea area and on the elementary sea patch size, a Bresenham algorithm is used for each transmitting and receiving antenna of the radar system in order to precompute the propagation plans needed to cover all the patches, while minimizing the total propagation distance.

\section{Antennas and targets configuration}

The antenna (or antenna array) locations of the radar system (monostatic or bistatic) are defined by the user. Associated radiation patterns or equivalent dipole sources model (linked to a database) will be available. The heading, velocity and type of vessel will also be specified by the user.

\section{Radar processing and visualisation}

From the "tree-view", different results corresponding to the radar processing defined at the beginning of the calculation will be available for checking: range processing by correlation with the transmitted signal, for each recurrence of the signal, thus leading to Recurrence-Range maps, Doppler processing performed on the previous image by Fourier transform of each recurrence, giving Range-Doppler maps, angular processing by digital beamforming if there are several reception antennas, providing complex Azimuth-Range-Doppler maps. These results will provide direct information on the detectability of the target(s) for the defined scenario

\section{THE ANTENNA MODEL}

The antenna module aims at providing the propagation tool with an incident field corresponding to the surface wave. It can be obtained either analytically or through a near field - far field calculation in the case of measurement data availability. The analytical calculation requires as an input a precise geometry of the antenna and the knowledge of the ground characteristics at its location. In addition, a list of typical HF antennas with pre calculated surface wave patterns is proposed to the user.

The modelling of the antennas in SimROS is performed through a model of the radiating structures obtained by means of a set of equivalent dipoles stored in a library [2]. This choice allows to pre-process the complete modelling of the antenna with its environment (beach, cliff...) outside the code in order to not degrade the computation time. The implementation of the dipole model can currently be done in two ways. The first one is based on data computed by means of one of the two following codes: the ICARE code of IEEA [3] which performs a resolution of integral equations associated with complex images theory to take the actual interface into account or the TEMSIFD software of Xlim based on the FDTD method [4]. The second way uses data measured under real working conditions. Both approaches make it possible to define the near field radiated on a virtual closed surface $S_{a}$ surrounding the antenna. It serves as a reference for the determination of the complex amplitude of the dipole moments located on a second virtual surface $S_{d}$, internal to the previous one. By default, the considered surfaces are cylindrical. By applying Bannister's analytical dipole radiation formulae, a matrix system can be built that relates the dipole moments to the fields radiated on the surface $S_{a}$. This system is oversized and is resolved by singular value decomposition (SVD). The use of the TSVD (Truncated SVD) associated with a power criterion makes it possible to limit the number of dipoles to those which are significantly contributing to the radiated field. In addition, the work carried out has shown that only the electric field components are required, assuming a plane wave relationship between the electric and magnetic field components. This latter assumption is particularly advantageous when the fields come from measurements. To illustrate the second method, we take the example of a 1/100 scale model of a biconical antenna, typical of HF radars, placed on a sandy soil. From the measurement of the near electric field components radiated by this biconical antenna, the dipole moments of the equivalent sources have been determined, solving the linear system previously mentioned. It is then possible to compute the far field at any greater distance outside $S_{a}$.

In Fig. 3, the amplitude of the vertical electric field (distance 3000ג@1GHz) radiated by the equivalent dipoles is compared to that obtained by a direct calculation using the commercial software FEKO. These first results, obtained in the UHF band, are very encouraging. A measurement campaign on $\mathrm{HF}$ antennas (scale 1) has been performed in autumn 2019 whose results will be presented during the conference.

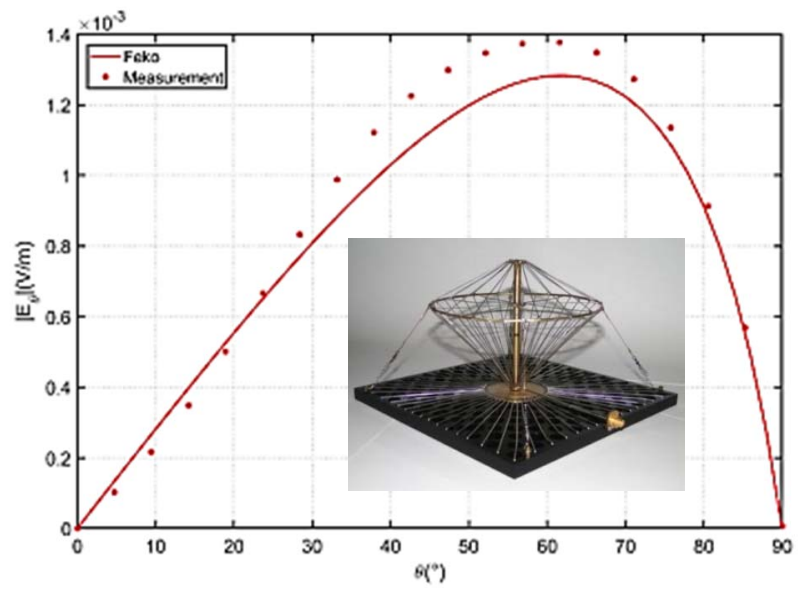

Fig. 3: Magnitude of the far electric field (distance 3000 $\lambda @ 1 \mathrm{GHz}$ ) comparison between equivalent sources model and FEKO software 


\section{SOLVER FOR SURFACE WAVE PROPAGATION OVER THE SEA}

The previous part was dedicated to the definition of an equivalent model of the antenna. Using the Bannister equations, the fields radiated by each elementary dipole are summed up to compute the near field which is used as the input of the propagation software. This toolbox aims at calculating the field attenuation on antenna - target links and antenna - patches links, these latter allowing to estimate the sea clutter noise. The propagation algorithm is based on the one-way (forward) resolution of the parabolic equation. The technique developed solves a 2D problem, marching on in space from the source location to a given observation point. The field is calculated on vertical lines with respect to the mean tangent plane. The ground wave radiated by the antenna can alternate propagation at the air - ground and at the air - sea interface. Particularly, it might happen that the link intercepts an island before reaching the vessel. The consistency of the results provided has been cross checked with respect to a classical problem, namely the one exhibiting the Millington effect.

Two approaches have been developed: the first one is based on the Finite Difference method and the second one on the Multiple phase (MPS) screens method. For the first one, a centred finite difference gives a second order accuracy but requires the inversion of a tridiagonal matrix. This is done using an algorithm implementing the Cholesky method. To correctly model the roughness of the sea surface, a conformal mapping strategy has been adopted. The consequence of this choice is a complexification of the equations but a resolution in a simple rectangular mesh. Moreover, a PML strategy has been used to avoid parasitic reflections on the topside of the computational volume. The MPS approach meets the Leontovich boundary condition at the interface. The sea state is characterized by a Pierson - Moskowitz spectrum. It is included in the algorithm as a modification of the impedance boundary condition. The wind speed at the interface scales into the sea state and to additional losses assimilating the waves on the sea to a rough surface.

The example considered is a ground - sea - ground - sea link. The wind direction makes a $45^{\circ}$ angle with the line of sight Fig. 4 shows the losses depending on the sea state. They may reach as much as $10 \mathrm{~dB}$ after propagation over $120 \mathrm{~km}$ between a sea state with no wind and a sea state corresponding to a strong wind. These losses are calculated at the ground (or sea) level.

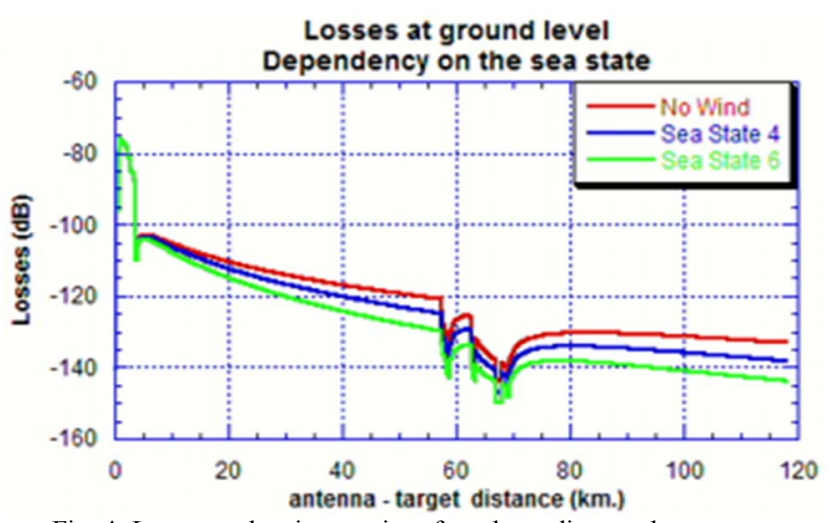

Fig. 4: Losses at the air - sea interface depending on the sea state

\section{RADAR CROSS SECTION (RCS) IN SEA ENVIRONMENT}

The objective of this part is to determine the RCS of maritime targets. The RCS calculation is an exact calculation, involving the Sommerfeld integrals evaluation as the sea surface is not perfectly conducting. The examples of RCS computation presented hereafter have been made using the ICARE software developed at IEEA [5] but they also can be made by a FDTD code. The objective is to build a RCS data base in surface wave mode, which can be used by the SimROS simulator.

There are two modifications with respect to the classical RCS calculation of an isolated target. The first one is related to the incident field and the second one to the calculation of the currents induced on the target considering the sea as a lossy medium. The incident field is the one obtained after propagation at the sea surface. Its dependency with the altitude above sea level is shown on Figure 5 corresponding to a propagation link over $120 \mathrm{~km}$.

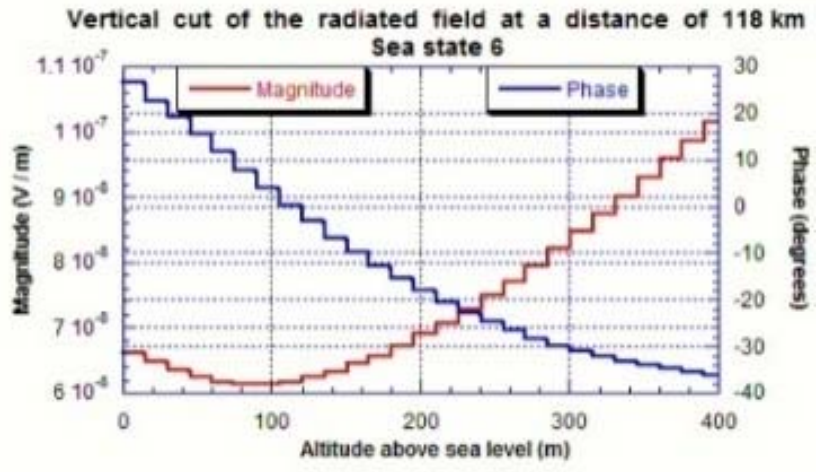

Fig. 5: Incident field at the target location

The RCS calculations hereafter presented have been obtained considering a frigate, approximately 100 meters long, as the scattering structure. The unitary incident field is along the roll axis. Figure 6 shows the difference that results of approximating the sea surface by a perfect ground plane. This result was obtained with the following hypothesis: when it is a perfect ground plane, the target image with respect to the sea surface is considered; when it is the real interface, the vessel immersed part of the structure is taken into account and Sommerfeld integrals calculation is involved.

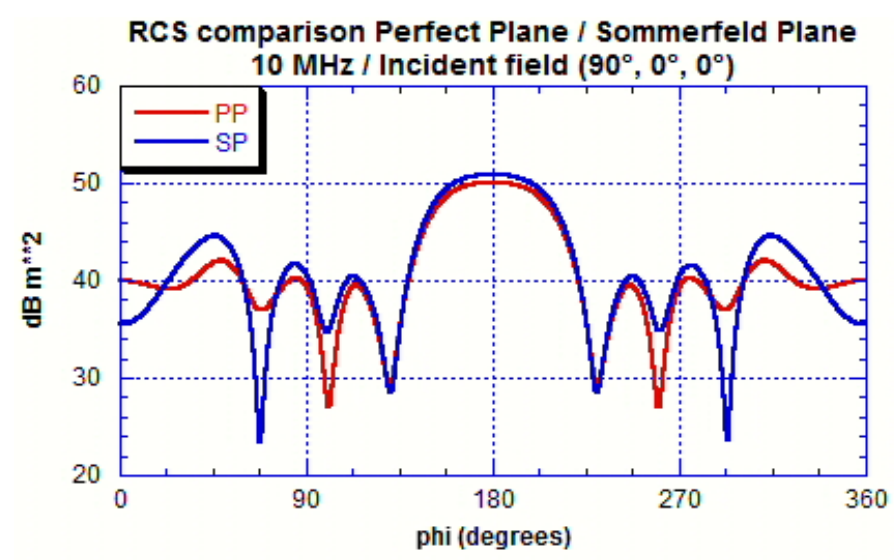

Fig. 6: Ground wave radar cross section of a frigate 
The results quite agree at high levels corresponding to the specular direction. They were obtained for an incident field not depending on the altitude. Taking the field variation as shown on Figure 5 into account does not change significantly the results as this variation with altitude is quite small in the HF band.

\section{RADAR LINK BUDGET VIA THE RECIPROCITY THEOREM}

The calculations are performed identically to evaluate the field and the noise contributions. As regards the field contribution, two links are considered. The first one is from the transmitting antenna to the ship and the second one from the ship to the receiving antenna. Use of the complex field allows summing the different contributions. As indicated previously, the transmitting antenna model inputs the parabolic equation in order to evaluate the field on the target. As the antennas are represented by dipoles models, it is not possible to use them directly in a reception mode. Instead, the problem is solved in transmission mode using the reciprocity theorem. For this purpose, the field due to the receiver in transmitting mode is calculated at the boat level. Use of the reciprocity theorem requires knowing the scattered field by the target. This field is the result of a bistatic RCS computation. The electric and magnetic equivalent sources used for reciprocity are deduced from this calculation. A scalar product is then used to obtain the voltage at receiver level.

This methodology also applies to the sea clutter noise calculation (diffraction by a sea patch illustrated in Fig. 7). The summation of complex voltages induced on the receivers allows obtaining the total sea clutter noise contribution.

\section{VII.RANGE DOPPLER PROCESSING}

As the software is currently under development, we do not have the possibility to present range/doppler results. One of the consortium partners, Onera, has made some preliminary experiments showing the kind of results we will get in the final version of the simulator. A typical example is presented in Fig. 8. These figures show a velocity - distance pattern obtained using a radar located in Biscarosse (France). This radar is considered in a monostatic configuration.

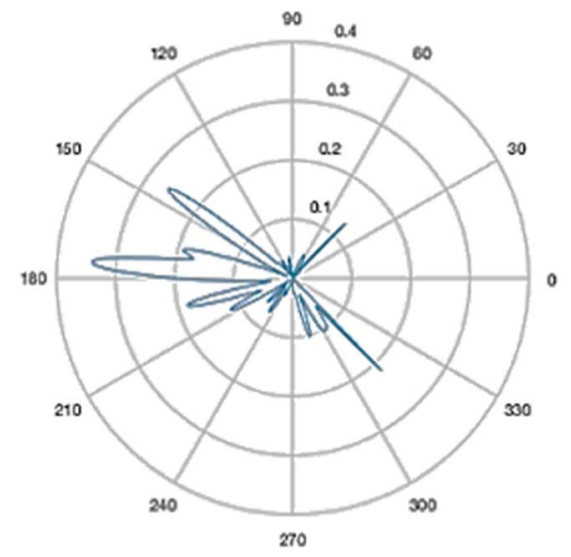

Fig. 7: Diffraction coefficient of a sea patch
The difference between the two graphs is the operating frequency. It shows a shift of the two Bragg lines that corresponds to $f_{B}=1.01 \sqrt{f_{0}(\mathrm{MHz})}$ where $f_{B}$ is the Bragg frequency and $f_{0}$ the operating frequency. The signal processing has been carried out on the corresponding data. Five different targets can be identified mainly located between the two Bragg lines.

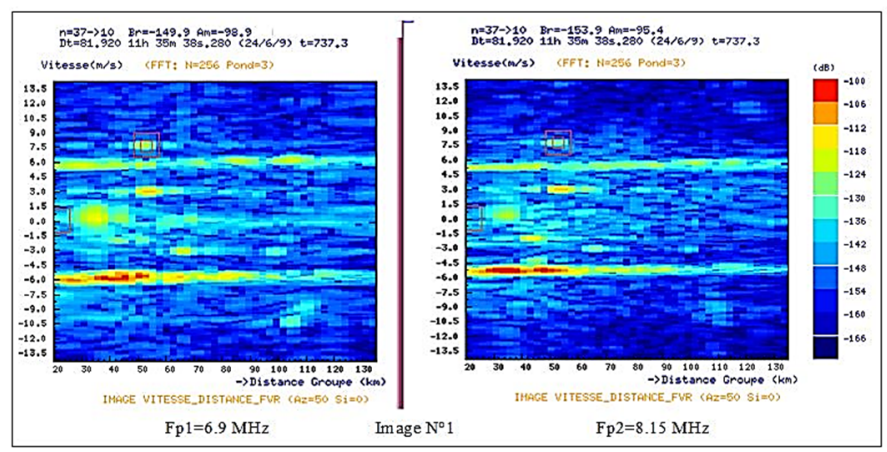

Fig. 8: Velocity - Doppler image for HF radar in a monostatic configuration

\section{CONCLUSION}

The main features of a numerical HF surface wave radar simulator, able to simulate the detection of targets far from the coast, have been presented. It is composed of a set of different toolboxes, each of them implementing state of the art resolution techniques, optimized for computational calculation in a reasonable time. This offers a large capability to users when intending to deploy such a radar to get an estimation of the expected performances. In a near future, the integration should have been completed and some results comparable to the experimental ones will be available.

\section{ACKNOWLEDGMENT}

The authors thank the AID of the DGA for its financial support via the ANR ASTRID Maturation SIMROS.

\section{REFERENCES}

[1] L. Sevgi and S. Sanal, "Surface wave HF radar simulator," Radar 97 (Conf. Publ. No. 449), Edinburgh, UK, 1997, pp. 181-184.

[2] N. Bourey, M. Darces, Y. Chatelon and M. Hélier, "Near-field to far-field transformation applied to UHF antennas over lossy ground.", International Journal of Microwave and Wireless Technologies, 1-6. doi:10.1017/S1759078719001272.

[3] http://www.ieea.fr/fr/logiciels/references/icare-mom.html

[4] Time Electromagnetic Simulator-Finite Difference Software, TEMSI-FD, CNRS, Univ. De Limoges, Limoges, France, 2006.

[5] RCS workshop at the European Defence Agency, Brussels, October 2019 